Facultad de Artes ASAB. Fotografía: Silvana Flores. Cortesia de la artísta






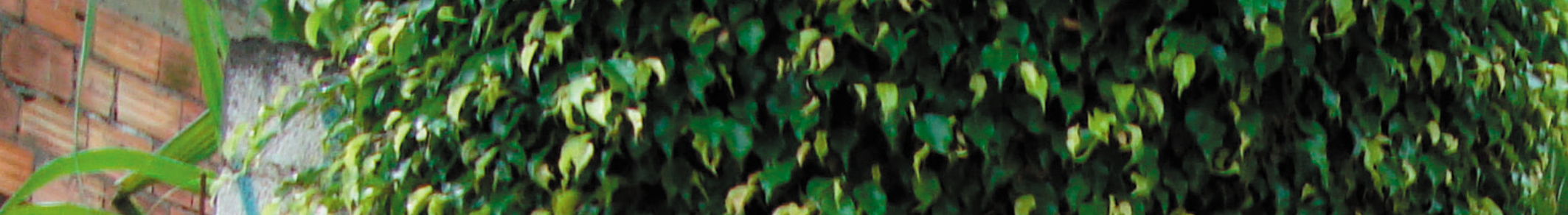

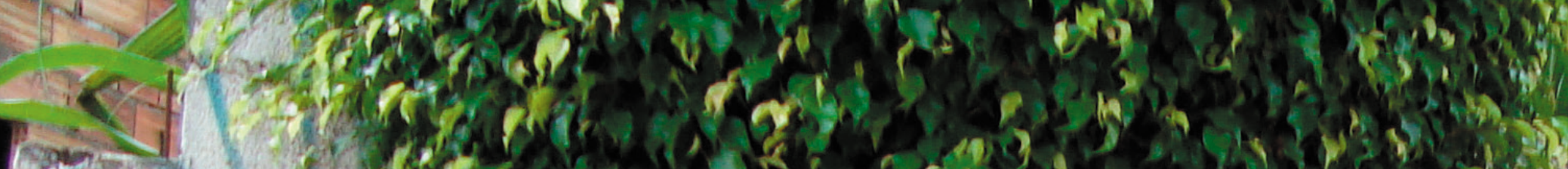

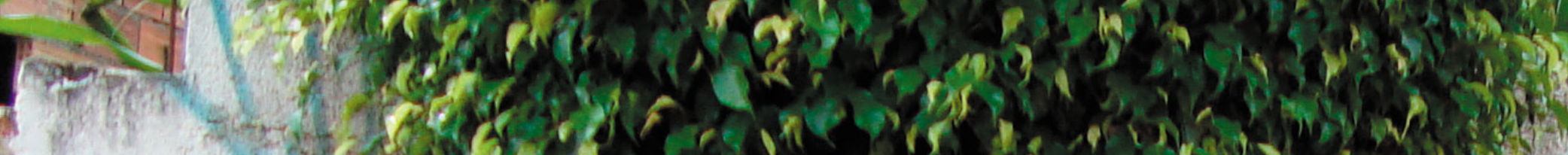

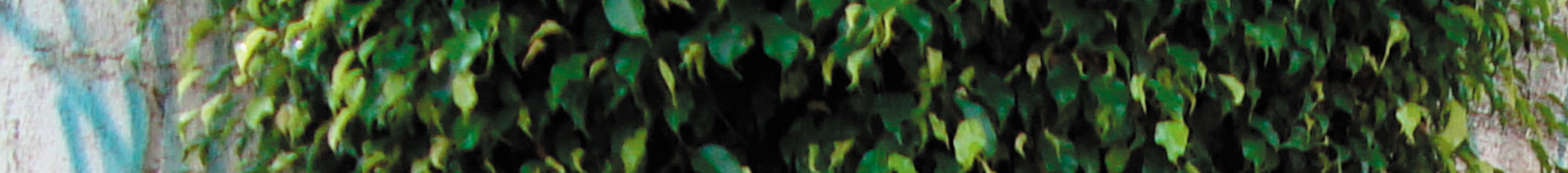

b)

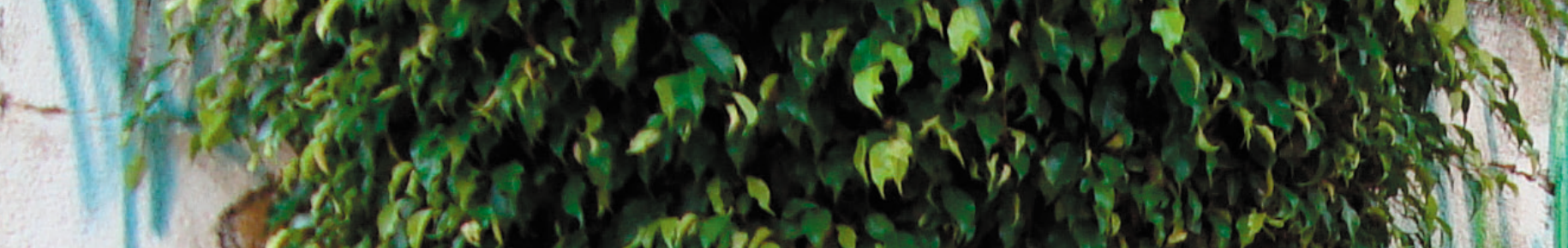






\section{VAI. Una apuesta por la cultura popular periférica emergente}

\section{Artículo de reflexión}

Estefanía González Vélez

Universidad Nacional de Colombia

estefania39@gmail.com

@1)(20

Cómo citar este artículo: González, E. [2017]. VAl. Una apuesta por la cultura popular periférica emergente, Calle14 revista de investigación en el campo del arte, 12[२2], 254-267

DOl: https://doi.org/10.14483/21450706.12357

\section{Resumen}

Este artículo hace parte de la tesis de doctorado "Tráficos culturales: la construcción de nuevas subjetividades en las periferias de Medellín y Sao Paulo", un estudio comparativo entre las dos ciudades a partir de la comprensión de las prácticas y políticas que se encuentran y articulan en sus periferias. La presente selección desarrolla la genealogía del programa de política municipal VAl - Valorización de las Iniciativas Culturales, y presenta sus mecanismos de trabajo, así como los ejercicios de apropiación por parte de los jóvenes ubicados en dichas áreas. Este programa se entiende como una herramienta que permite el surgimiento de jóvenes, que contribuye a la consolidación de un movimiento urbano y aporta a una nueva definición de la periferia y a la construcción de nuevas subjetividades a partir del campo cultural.

\section{Palabras claves}

Juventud, Medellín, movimientos, periferia, política cultural, Sao Paulo. 


\section{VAI. A Bet for Emerging Peripheral Popular Culture}

\section{Abstract}

This article is part of the doctoral thesis "Cultural Traffics: The Construction of New Subjectivities in the Peripheries of Medellin and San Pablo", a comparative study between the two cities based on the understanding of the practices and policies that are found and articulated in their peripheries. The present selection develops the genealogy of the municipal policy program VAI - Valuation of Cultural Initiatives - and presents the intricacies of its work, as well as the appropriation exercises by the young people who live in those areas. This program is understood as a tool that allows the emergence of young people, which contributes to the consolidation of an urban movement and to a new definition of the periphery and the construction of new subjectivities from the cultural field.

\section{Keywords}

Youth, Medellin, movements, periphery, cultural politics, Sao Paulo.

\section{VAI. Un pari pour la culture populaire périphérique émergente}

\section{Résumé}

Cet article fait partie de la thèse de doctorat « Trafic culturel : la construction de nouvelles subjectivités dans les périphéries de Medellín et San Pablo », une étude comparative entre les deux villes en fonction de la compréhension des pratiques et des politiques trouvées et articulées dans ses périphéries. La présente sélection développe la généalogie du programme de politique municipale VAI -Évaluation des initiatives culturelles - et présente ses mécanismes de travail, ainsi que des exercices d'appropriation par les jeunes situés dans ces domaines. Ce programme est compris comme un outil qui permet l'émergence des jeunes, qui contribue à la consolidation d'un mouvement urbain et à une nouvelle définition de la périphérie et à la construction de nouvelles subjectivités dans le domaine culturel.

\section{Mots clés}

Jeunesse, Medellin, mouvements, périphérie, politique culturelle, Sao Paulo.

\section{VAI. Uma aposta pela cultura popular periférica emergente}

\section{Resumo}

Este artigo faz parte da tese do doutorado "Tráficos culturais: a construção de novas subjetividades nas periferias de Medellin e San Pablo, estudo comparativo entre as duas cidades a partir da compreensão das práticas e políticas que se encontram e articulam nas suas periferias. A presente seleção desenvolve a generalogia do programa de política municipal VAl- Valorização das iniciativas Culturais, e apresenta seus mecanismos de trabalho, assim como os exercícios de apropriação por parte dos jóvens localizados em ditas áreas. Este programa se entende como uma ferramenta que permite o surgimento de jovens, que contribui à consolidação de um movimento urbano e aporta a uma nova definição da periferia e à construção de novas subjetividades a partir do campo cultural. 


\title{
Palavras-chave
}

Juventude, Medellín, movimentos, periferia, política cultural, San Pablo.

VAI. Sug apuesta cultura popularmanda periferika emergente.

\section{Maillallachiska:}

Kai articulok kame parte sug tesis de doctorado "tráficos culturales: sug construcción musu subjetividades periferiakunape Medellinpe y San Pablope", chi estudiok comparativo iskandi ciudadkunape a partir comprensionmanda chi prácticas y políticas kankuna y articulan paipa periferiakunape. Kai presente seleccionka desarrollame sug genealogía del programa sug política municipal VAlValorización de las iniciativas culturales y kauachi paipa mecanismo de trabajo, chasallata ejerciciokuna de apropiación musukuna ubicado chi areakunape. Kai pregramak entendereme sug herramienta permitime llugsingapa musukuna, contribuyenkuna a la consolidación sug kuiurii urbano y aportarme sug musus periferia y uiñachii musu subjetividad a partir del campo cultural.

\section{Rimangapa Ministidukuna:}

Musukuna, Medellin, kuiuriikuna, periferia, política cultural. Sao Paulo

Primero llegaron los colectivos, después llegó VAl.

\author{
James Lemos Abreu
}

El Programa Valorización de Iniciativas Culturales - VAl, implementado por la Secretaría de Cultura Municipal de São Paulo, es una política pública que subsidia financieramente, hasta por dos años, proyectos en diferentes lenguajes artístico-culturales, desarrollados por personas de estratos bajos, especialmente jóvenes entre los 18 y 29 años. ${ }^{1}$

"Se destacan dos aspectos peculiares del programa, y que tienen estrecha relación entre sí, a saber: el reconocimiento de las acciones culturales juveniles en desarrollo en las áreas periféricas de la ciudad y, consecuentemente, una concepción más amplia de la pluralidad de las prácticas artísticas y culturales realizadas" [Secretaría de Cultura, 2012: 15].

El análisis que se presenta a continuación profundiza en el conocimiento y comprensión de esta política pública como una herramienta para los jóvenes dentro del escenario contemporáneo de la nueva democratización cultural de nuestras ciudades. Es importante aclarar que todo lo que tiene que ver con las descripciones y análisis de este programa está ubicado temporalmente entre el surgimiento del mismo y el final del año 2012. En el año 2014 fue aprobado en el Consejo la sanción para el Proyecto VAl 2.

Más allá del interés por conocer la política en detalle, prevalece el de comprender los modos como son apropiadas este tipo de políticas por parte de los ciudadanos, el uso que hacen de ellas, la manera como se inscriben en el campo cultural por medio de las mismas,
1 En la descripción del Programa VAl, se explica que la finalidad del mismo es apoyar financieramente, por medio de subsidio, actividades artístico -culturales principalmente de jóvenes de estratos bajos en las regiones desprovistas de recursos y equipamientos culturales. Si bien las convocatorias y el programa mismo no precisan que los recursos sean exclusivos para la periferia, en el imaginario común estos lugares se comprenden como tal. 
los impactos que producen en su vida cotidiana, las relaciones que establecen y los valores políticos ligados a la participación, entre otros.

Se ha tomado el caso de VAl con el ánimo de comprender la capacidad de generar articulaciones y relaciones entre los jóvenes y el gobierno mediante políticas públicas municipales, sectoriales, segmentadas para jóvenes de estratos bajos en el ámbito de la cultura, así como las posibilidades de empoderamiento y emancipación que estos dispositivos permiten, al reconocer unas identidades culturales bastante específicas. Del mismo modo, se intentará analizar si es posible que el enriquecimiento del campo cultural dado por el reconocimiento estatal de estos nuevos sujetos [traficantes culturales, jóvenes de periferia], por medio de las políticas públicas, produzca impactos positivos en la democracia y la construcción de una ciudadanía activa.

Los orígenes del Programa VAl se remontan a las discusiones de la Comisión Extraordinaria y Permanente de Juventud del Consejo Municipal de São Paulo, desarrolladas entre 2001 y 2002, en la que participaron parlamentarios de diferentes partidos, así como movimientos de la sociedad civil. La comisión fue dirigida por el Concejal Nabil Bonduki $^{2}$ del Partido de los Trabajadores, quien siempre había contado con el apoyo de la juventud, especialmente de clase media; la apropiación del Programa VAI por parte de los jóvenes inicia con su propia creación, ya que de acuerdo con el testimonio de los gestores de la política, esta surgió de la demanda de los jóvenes "desde las bases"3. $^{\text {. }}$

A pesar de que la creación del Programa se relaciona con las coyunturas propias de la época, fue a partir de

2 En febrero del año 2015 Nabil Bonduki tomó posesión como Secretario Municipal de Cultura de São Paulo, nombrado por el alcalde Fernando Haddad. Bonduki sustituyó a Juca Ferrerira, quien asumió el Ministerio de Cultura. El exconcejal fue relator del Plan Director de Cultura de la ciudad y de un conjunto de directrices para la planeación del crecimiento de São Paulo en los próximos 16 años; así mismo, fue uno de los ideólogos del Programa VAl, un programa de estímulos para iniciativas culturales juveniles de la periferia. [Recuperado de: http:// www.icarabe.org/noticias/nabil-bonduki-toma-posse-comosecretario-municipal-de-cultura-de, el 23 de agosto de 2015]. 3 Durante los meses de agosto a diciembre de 2012 se realizaron diferentes entrevistas en la ciudad de São Paulo con la directora del Programa, María do Rosario Ramalho, el coordinador, Gil Marçal, las investigadoras y miembros de la comisión evaluadora Ana Paula do Val y Rita Alves, el técnico que acompañó el proceso de la Agencia Popular, Solano Trindade James Lemos Abreu, entre otros miembros activos o fundadores del programa. Así mismo, se hizo acompañamiento de algunas de las visitas técnicas realizadas durante este periodo a diferentes movimientos beneficiados por VAl. las discusiones de dicha comisión que empezó a surgir la propuesta de crear una política pública de cultura para jóvenes, aprovechando un período en el que se acrecentó el interés por comprender mejor el papel que la cultura estaba teniendo en la vida de los jóvenes paulistas. Se trataba de un contexto político favorable en el que la comisión tenía la posibilidad de ampliar y profundizar en la discusión sobre la cuestión de la juventud como interés de las políticas públicas, para responder a preguntas como: ¿Qué joven es este? ¿Cuáles son sus deseos? ¿Cuáles son sus necesidades? ¿Cómo pueden las políticas ayudar a resolverlos y alcanzarlos?

Este momento histórico estaría, además, marcado por una transición en el gobierno local, pasando de dirigencias marcadamente conservadoras a una administración que tenía como bandera la renovación de las políticas públicas en cabeza de Marta Suplicy [2001-2004], del Partido de los Trabajadores. La voluntad de los cambios se confirmaría en 2003 con el triunfo de Luiz Inácio Lula da Silva, también del PT, en el gobierno federal. Para el Partido de los Trabajadores resultaba fundamental el rol de la cultura en el desarrollo de una sociedad más democrática; en este sentido, podría decirse que era el momento preciso para la creación de propuestas tan arriesgadas, innovadoras y populares, como VAl [municipal], ProAc [estadual/ departamental] o Cultura Viva [Nacional].

\section{Si bien es cierto que la Comisión Extraordinaria y}

Permanente de la Juventud buscaba abordar diferentes asuntos como educación, trabajo, salud, etc., hay que decir que uno de los temas más destacados y recurrentes en las discusiones fue el de la cultura, normalmente relegado. Este tema fue cobrando relevancia, especialmente a medida que los jóvenes fueron participando de las reuniones de la comisión. Se realizó un importante ejercicio de motivación para vincular a los jóvenes en las discusiones, lo que sería determinante en el carácter cultural del proyecto de ley propuesto. Los debates resaltaron la importancia de la cultura para la vida de los jóvenes, para su ocio y sociabilidad, y, al mismo tiempo, como base para sus experiencias en el proceso de definición de la identidad, la construcción de referencias y visiones del mundo, así como para la expresión de estas y, en este sentido, para su participación social. "Los jóvenes señalaron la falta de equipamientos, apoyo y recursos para posibilitar la vivencia cultural que demandaban, tanto en el sentido del consumo [goce] como en de la producción de cultura" [Secretaría Municipal de Cultura, VAl - 5 años, 2008].

Esta serie de inquietudes y demandas coincidía con el surgimiento de un movimiento social que le confería 
una mayor visibilidad a la juventud en el ámbito del gobierno estadual y municipal por medio de la creación de secretarías y grupos de trabajo, orientados específicamente a este segmento de la población. Tal es el caso de la Secretaría de Estado para la Juventud, creada en 1999 mediante la ley 10.387, y la Coordinadora Especial de Juventud del Municipio de São Paulo, creada en 2001 mediante la ley 13.169. Pero, más allá de esto, la preocupación por la cuestión de la juventud, envolvía un movimiento que traspasaba las fronteras del Estado y vinculaba una serie de actores de la sociedad civil, entre los que se destacaban algunas ONGs con gran influencia en la ciudad como El Instituto Pólis ${ }^{4}$ o la Acción Educativa ${ }^{5}$, entre otras. Desde este movimiento comenzaron a producirse estudios e investigaciones que ayudaron a reforzar la importancia de la cuestión de la juventud en la agenda política y que alcanzaron bastante influencia en las agendas públicas.

Estudios como la "Investigación sobre la juventud: cultura y ciudadanía", del 2000, realizada por el Núcleo de Opinión Pública [NOP] de la Fundación Perseu Abramo ${ }^{6}$, y el "Mapa de la Juventud de la Ciudad de São Paulo", de 2003, elaborado por la Coordinadora de Juventud de São Paulo, permitieron romper varias representaciones comúnmente asociadas con la juventud y reforzadas incesantemente por los medios, como la falta de interés por la cultura, la relación estrecha con la violencia y la rebeldía "sin causa", revelando en cambio el perfil de un joven curioso, preocupado con cuestiones públicas, y ávido en producir cultura y participar de iniciativas existentes en su propia comunidad. Así pues, más allá de una visión estereotipada de la condición juvenil, se rescataba su condición de ciudadano, de sujeto de derechos [Secretaría Municipal de Cultura, VAl - 5 años, 2008). El estudio de la Fundación Perseu Abramo fue material fundamental para definir los temas prioritarios de los debates que se desarrollarían con los jóvenes en el Consejo. Salud, educación, sexualidad y seguridad, fueron temas recurrentes en el estudio. Pero, además de estos, la cultura aparecía a lo largo de la investigación como un tema clave y estructurador para la juventud: para actuar, socializar, experimentar y participar. A partir de esto, se propuso un debate en el que participaron personas conocedoras del tema de la juventud y la cultura brasileñas como Helena Abramo, Luciana Guimmaraes y Maria del Rosario Ramalho, entre otros, quienes además de conocer ampliamente estos temas, habían participado en diferentes espacios del gobierno municipal o nacional. Se desarrollaron interesantes debates durante un año, que se encuentran consignados en la publicación "Un año de Juventud" [Comissão Extraordinaria Permanente da Juventude, 2012].

Adicional a los debates, se realizaron visitas de campo a la periferia, así como a otros municipios que venían aplicando programas de democratización de los recursos, como el presupuesto participativo o las leyes de incentivos. Ante la gran demanda que encontraron entre los jóvenes de las periferias, por contar con recursos para realizar sus propias producciones artísticas, y teniendo en cuenta los aprendizajes de otras políticas, comenzó a gestarse una primera propuesta de proyecto de ley para financiar las iniciativas de colectivos culturales.

La mayoría [de los jóvenes] o ve televisión o juega fútbol en pequeños campos [...] porque no tenemos muchas canchas, como se puede ver [...] pero ellos no ven televisión porque les gusta; ven porque no tienen otra cosa para hacer, no tienen otras actividades [...] lo que a ellos les gustaría hacer es estar en un parque, en un club polideportivo desarrollando sus actividades, en un centro cultural fomentando sus actividades, sus talentos" [Valdeir do Santos Pereira, 16 años] [Comissão Extraordinaria Permanente da Juventude, 2012]

La primera opción para responder a estas demandas fue replantear la ley de incentivo. Sin embargo, la gran dificultad que se ha experimentado con esta política es que a las empresas del sector privado no les interesa entregar recursos para proyectos de periferia o cultura popular. En este sentido investigadores como Isaura Bothelo han dado un gran debate, argumentando que la producción cultural no puede estar determinada por los intereses de las grandes empresas, como viene ocurriendo a partir de la implementación de estas leyes. Si bien son válidas, y generan un aporte importante en el desarrollo cultural del país, las políticas culturales no pueden reducirse a esto [2001]. Como contrapropuesta se planteó que un porcentaje de lo que se recaudaba por ley de incentivo fuera 
para proyectos de jóvenes de las periferias; no obstante, la propuesta no fue bien recibida por los productores culturales que captan los recursos de la empresa privada y no fue comprendida por los jóvenes de las periferias. Los artistas y productores consagrados consideraban injusto que además de tener que gestionar sus recursos, tuvieran que gestionar los de las periferias; los jóvenes periféricos no le encontraban sentido a contar con lo que sobrara de los grandes proyectos.

Recorrimos las diferentes zonas de la ciudad presentando el proyecto. Las personas no lo entendían, no encontrábamos respuesta. Ahí fue que pensamos "algo anda mal", si la gente no comprende, no va a participar, no habrá apropiación. Y de nuevo empezamos a pensar; teníamos que llegar a un proyecto de ley que fuera mucho más simple para los jóvenes. De ahí nació la idea de lo que se convertiría en VAl. [María do Rosario Ramalho, 2012]

Los primeros borradores del proyecto se pensaron para organizaciones juveniles; sin embargo, pronto se dieron cuenta que las formas de asociación de la juventud hoy pasan por la informalidad, así que este era un principio excluyente. Los jóvenes paulistanos se estaban uniendo por causas y proyectos que a veces permanecían poco en el tiempo, pero otras veces se disolvían una vez desarrollaban las acciones. Así que crear el programa necesitó un profundo trabajo de campo que permitió comprender mejor a la juventud paulistana entendiendo que era necesario crear un dispositivo legal que al mismo tiempo ampliara las condiciones de acceso a la cultura y simplificara las exigencias para participación en convocatorias y procesos selectivos. Esto explica porque VAl está dirigido a personas naturales [aunque también recibe personas jurídicas], jóvenes entre 18 y 29 años [un rango de juventud más amplio que el que ha reconocido las Naciones Unidas] de la periferia que realizan prácticas culturales. De acuerdo con los integrantes de la comisión, más allá de la edad, el programa debía considerar otros aspectos relacionados con la condición juvenil, ligados específicamente a su búsqueda de autonomía, reconocimiento e identidad. De acuerdo con Luciana Guimarães quien integró la Comisión Coordinadora de la Juventud:

Esta es la fase de la construcción de referencias que determinan elecciones de los y las jóvenes para entrar en el mundo adulto; es también un momento en el que se da el desarrollo de la autonomía. Por eso, las acciones pensadas para esta población, deben siempre procurar, por un lado, entender el repertorio de los jóvenes, garantizando así que las elecciones puedan ser hechas, y por otro, proporcionar experiencias de autonomía. [Guimarães en De Lemos, 2010: 144]

Terminado el 2002, se había consolidado un proyecto de ley pensado con y para los jóvenes de la periferia. El año 2003 constituyó un momento definitivo para la implementación de VAl. La Secretaria Municipal de Cultura, por medio del Departamento de Teatro, enfrentó el desafío de la institucionalización del programa, que vendría a financiar prioritariamente personas naturales, de una forma completamente diferente a la que se había utilizado en aquel organismo hasta entonces.

\section{La puesta en marcha}

La "jugada política" que permitió sacar adelante esta propuesta tan arriesgada fue presentársela primero al Secretario de Cultura Celso Frateshci, quien se comprometió a implementarla si pasaba como ley. Cuando un proyecto recorre el camino normal de postulación para ser ley y no pasa antes por la "pre-aprobación" (informal) de un funcionario público que pueda ponerlo en marcha, las posibilidades de que pase son pocas; se puede caer en el camino o tardar años en ser aprobado. Digamos que el ejecutivo no permite que el legislativo actúe sin antes consultarle, explicó un funcionario. Así que, una vez fue conocido en la Secretaría de Cultura y pasó los debates en el Consejo, el proyecto fue aprobado como ley municipal 13.540 en marzo de 2003. En septiembre de ese mismo año la ley fue reglamentada por la alcaldía mediante el decreto 43.823 y, un mes después, se hizo la primera convocatoria, en la que participaron 650 proyectos. Para diciembre ya se habían elegido los primeros 67 proyectos beneficiados. Fue un momento bastante bueno para VAl: comenzó a ser visible y reconocido entre los jóvenes de la ciudad, en gran medida gracias a la divulgación institucional y al reconocimiento de organizaciones que actuaban en el campo de la inclusión social de los jóvenes, que se encargaron incluso de promover talleres de elaboración de proyectos para ser presentados al programa. La realización de estos cursos y de un material didáctico generado por el propio programa (Agora VAl: Roteiro para elaboração de projetos culturais como foco no Programa VAl], fueron fundamentales para la ampliación del público de VAl. En los primeros años VAl se desarrolló con recursos de reserva, posteriormente se le asignó un presupuesto anual.

Los 67 proyectos financiados en el 2004 recibieron los recursos en tres desembolsos, de acuerdo con el cronograma de actividades de cada uno; estos desembolsos estaban supeditados a la entrega de informes y presentación de cuentas. Para ese momento el 
programa contaba con una coordinadora y dos personas administrativas, quienes además respondían por funciones del Programa de Fomento al Teatro. Para la selección de los proyectos se contó desde el principio con la Comisión de Evaluación, un grupo compuesto por funcionarios del gobierno municipal y de la sociedad civil, que ha sido fundamental para determinar el desarrollo de VAl en cuanto a la línea de elección de los proyectos. Son las iniciativas beneficiadas las que le han dado el carácter al Programa. En un principio, los funcionarios que integraron la Comisión eran propuestos por el Consejo Municipal de Cultura, cada uno de ellos participaba como evaluador durante un año. A partir del 2005. la comisión pasó a ser escogida por la Secretaría de Cultura, entre las entidades inscritas por el Consejo de Cultura, de acuerdo con la ley, "porque simplemente la nueva administración en cabeza del alcalde José Serra, no volvió a convocar al Consejo Municipal de Cultura", según explicó James Lemos Abreu. Así mismo, la duración de cada miembro en la comisión pasó a ser de dos años y las personas serían renovadas de forma alternada de tal manera que siempre hubiera personas antiguas y nuevas. De acuerdo con el equipo técnico de VAl, esto ha sido muy positivo en la medida en que se mantiene una memoria de los procesos de elección y los debates ocurridos a lo largo del desarrollo de los proyectos; pues, además de seleccionar las iniciativas, la comisión se reúne a largo del año para evaluar el desarrollo del Programa.

La selección para los proyectos de la segunda edición ocurrió a finales de 2004, coincidiendo con un cambio de administración. Se inscribieron 450 proyectos y fueron seleccionados 71. El año 2005 fue determinante en cuanto a la consolidación del programa en el campo de las políticas públicas. El programa pasó del Departamento de Teatro al Departamento de Acción Cultural Regionalizada [posteriormente, pasaría al Departamento de Expresión Cultural donde permanece hoy]. Además, se contrató un técnico, y el equipo, aunque pequeño, quedó encargado solamente de VAl, desligándose de las responsabilidades de Teatro.

Al mismo tiempo, en el año 2005, cuando ocurrieron todos estos cambios positivos, el programa comenzó a tener una dirección propia con María del Rosario Ramalho [quien había participado desde los inicios en el diseño del mismo]. Pero, a su vez, el gobierno municipal entró en crisis y el presupuesto para cultura fue recortado en un 31\%. Los \$R16 mil [8.000 US aproximadamente] que eran asignados a cada proyecto elegido serían reducidos a 11 mil. Algunos jóvenes firmaron un acuerdo aceptando el recorte; sin embargo, la mayoría no lo aceptó y el programa se paralizó. La movilización llevada a cabo por los jóvenes agravó la crisis de la Secretaría a tal punto que el secretario fue cambiado por Carlos Augusto Calil, a quien los jóvenes lograron convencer de descongelar un recurso y mantener el monto de VAl en \$R16 mil. Las demoras en el desembolso del dinero obligaron a modificar los cronogramas y la metodología para el desarrollo de los proyectos ese año. En ese momento se consolidó aún más el equipo con la coordinación de Gil Marçal: su entrada al programa sería determinante en el futuro del mismo. Gil hacía parte de ese movimiento cultural de la periferia sur que comenzó a demandarle al Estado recursos para realizar sus prácticas. Su coordinación [2005-2012] fortaleció la confianza y la relación de los jóvenes con el Programa.

A partir del año 2005, se realizaron talleres de elaboración de proyectos en alianza con la Coordinadora de la Juventud, el Centro Cultural de la Juventud, y por medio de una entidad contratada por el Departamento de Expresión Cultural específicamente para esto. Estas estrategias, más allá de ampliar el público, comenzaron a darle pistas al equipo técnico y a la comisión de evaluación sobre las principales tendencias y perfiles de los proponentes. De alli surgió un especial interés por conocer mejor a los colectivos que presentaban los proyectos, su origen, sus búsquedas. A partir de esto, se empezaron a generar desde el 2005 estadísticas levantadas anualmente que dan cuenta del perfil de los grupos, las zonas de donde provienen, los lenguajes de mayor demanda, entre otros.

En el año 2006 se recibieron 758 inscripciones para 62 proyectos seleccionados. El equipo creció con un nuevo técnico y un practicante. Esto, sumado a un aumento presupuestal que pasó de $\mathrm{R} \$$ 1.000.000 a \$R 1.710.000, permitió un aumento del 62\% en proyectos seleccionados en 2007 , cuando de 777 que se presentaron se eligieron 102. Para el año 2008, el Programa contaba con una coordinadora, cuatro técnicos, dos practicantes y un presupuesto de $\mathrm{R} \$$ 2.000.000; se recibieron 705 inscripciones y fueron seleccionadas 115 iniciativas. Adicional a esto, desde ese año se cuenta con un blog que permite la comunicación con los grupos ${ }^{7}$. Año tras año ha venido aumentando la cantidad de proyectos seleccionados y el equipo se ha consolidado; en el año 2012 [año en el que se hizo esta investigación] el Programa contaba con una directora, un coordinador, seis técnicos, un administrativo y un practicante, y logró apoyar

7 Recuperado de: http://www.programavai. blogspot.com/, y la difusión permanente de información. , el 12 de febrero de 2015. 
178 proyectos por valores hasta de \$R23.000. Desde su creación hasta el año 2012, el Programa VAl había subsidiado 956 proyectos, entre personas naturales y jurídicas, con una inversión total de $\mathrm{R} \$ 18$ millones.

\section{La apropiación de un programa de política pública}

Uno de los mayores logros del Programa VAl es que ha colaborado en la ampliación de la oferta cultural de la periferia. Si bien es cierto que São Paulo es una ciudad con una gran oferta cultural, esta se concentra en el centro de la ciudad y resulta muy costosa y ajena para las personas que vienen de zonas alejadas y que son consideradas de estratos bajos. Lo que ha favorecido VAl es la puesta en marcha de prácticas culturales y la generación de un circuito permanente de acciones en diferentes zonas de la periferia ${ }^{8}$. Ahora bien, no se trata de decir con esto que fue el Programa quien creó las prácticas. Por supuesto que no, simplemente las reconoció y al entregar los recursos facilitó su realización. En la periferia existen un sinnúmero de prácticas anteriores a VAl como el Sarau da Binho, el Sarau de la Cooperifa, el Instituto Pompas Urbanas, el colectivo Imargen, entre otros, que son proyectos con larga trayectoria, creados por actores culturales que de alguna manera han construido una imagen y una propuesta propias de estas regiones. Sin embargo, el número de iniciativas resultaba poco en relación a las dimensiones de la periferia paulistana y las posibilidades de movilidad. Más allá de eso, hacían falta estrategias de articulación que permitieran crear circuitos y, de alguna manera, consolidar esa cultura periférica. Cabe decir que en gran medida fueron los proyectos existentes los que motivaron, junto a los debates, la creación de un dispositivo como VAl, así como la multiplicación de colectivos y acciones culturales por parte de otros jóvenes en las periferias. Es evidente que primero fueron los colectivos y después vino VAl. No obstante, la escasez de recursos limitaba mucho las prácticas y, como tantas veces lo han dicho los mismos jóvenes, la obligación de desarrollar actividades que les representen ingresos para su vida cotidiana, impedían que pudieran dedicar tiempo a sus prácticas, incluso parcialmente.

8 Es posible asistir a saraus los 7 días de la semana; hacia el final de la semana se presentan obras de teatro; los domingo se realizan eventos culturales como bingos, bazares, ferias de arte y cultura; constantemente se realizan conciertos, performances, muestras de cine entre otros. Se puede consultar la Agenda Cultural de la Periferia, elaborada por la ONG Açao Educativa: http:// www.agendadaperiferia.org.br.
La verdad es que aprendí que el arte en Brasil es complicado para sobrevivir. Cuando descubrí eso, me desilusioné mucho, y lo intenté resolver. Fui a ganar dinero. Una parte de mi tiempo intento ganar dinero y otra parte intento producir cosas que me gustan. Y una vez más percibo que el tiempo del negocio se me está comiendo el tiempo de lo artístico [joven de 31 años; participó en la convocatoria 2006-2007]. [Secretaria Municipal de Cultura, Via VAl: percepções e caminhos percorridos, 2012: 31].

En este sentido, la posibilidad que ha dado VAl de financiar acciones culturales propias les ha permitido a los grupos desarrollar las prácticas y, por medio de estas, tomarse, de una parte, los equipamientos existentes [centros culturales, bibliotecas, centros integrados, etc.] y, de otra, apropiarse de los espacios públicos como los bares, los parques y las plazas, consiguiendo con esto consolidar una producción cultural y simbólica de la periferia para la periferia. Esto ha conducido a la activación de un circuito cultural de las zonas más alejadas, lo que ha sido fundamental para vencer un obstáculo que deben enfrentar los artistas y productores culturales de la periferia: la formación de públicos. Se trata de un círculo, en la medida en que hay una mayor demanda y apropiación de la cultura periférica por parte del público, hay una multiplicación también de la oferta. No obstante, vale aclarar que esta circulación continua siendo zonal.

En la periferia... en los edificios en los que nos presentábamos, 90\% de las personas que venían a nuestra presentación, a una pieza de teatro, nunca fueron al teatro, nunca asistieron a una pieza, nunca... Estaban siempre encerrados viendo la televisión. Se limitan a la televisión [...] cuando usted iba dentro de un edificio, dentro de las casas de ellos, dentro del espacio de ellos y cuando las personas lo veían tenían miedo: "los artistas". Tenían hasta recelo de la persona que está pintada, de un artista, pero después de un cierto tiempo, fueron viniendo y veían que ese artista es una persona como ellos, de carne y hueso, una persona que tiene sentimientos, que intercambia ideas, que conversa, que es normal. Que no hay diferencia entre quien está en la televisión y quien está frente a usted [Secretaría Municipal de Cultura, 2012].

Por otro lado, en la medida en que el programa ha contribuido a conseguir los recursos necesarios para las prácticas culturales [equipamientos, vestuario, tecnología, etc.], se está contribuyendo a romper el prejuicio que existe sobre la calidad de la producción de la periferia. Es decir, entre los artistas consagrados que tienen acceso a otros estímulos, así como entre los financiadores que 
apoyan proyectos a través de la ley de incentivo, existe la idea de que todo lo que se hace en la periferia carece de calidad estética. Se tiene la idea de que esta producción es mediocre, "demasiado popular". De alguna manera, iniciativas apoyadas por VAl que se están desarrollando con un concepto estético han ayudado un poco a modificar esta noción. Sin embargo, hay que decir que este logro sigue siendo muy incipiente. De una parte, el prejuicio permanece y, de otra, la calidad estética sigue siendo muy cuestionable. En este aspecto tienen mucho que ver, de forma negativa, las mismas políticas de financiación que promueven propuestas artísticas sin exigencias de calidad, simplemente para cumplir con la asignación de un recurso y las cuotas de inclusión. Con lo que consiguen, además, generar una especie de "oferta propia", muy económica, que ponen a disposición del pueblo. Es decir, como se menciona en el caso de Medellín, se trata de llenar de contenido sus agendas culturales casi con el mismo recurso que se responde a las demandas de los jóvenes. El Estado financia proyectos culturales y el Estado los contrata por muy bajos costos, para sus propios eventos, supuestamente con el fin de generar circulación, pero sin competitividad; por esto, cuando pasan a escenarios del mercado, se quedan por fuera y se les juzga por la falta de calidad.

En este orden de ideas, y a pesar de ser un programa de cortos alcances ${ }^{9}$, puede decirse que VAl, como política pública, ha logrado el reconocimiento y la apropiación por parte de algunos jóvenes de las periferias que están encontrando en él la primera posibilidad de acceder a recursos públicos. Para muchos de ellos, que nunca habían realizado ejercicios de gestión cultural, su participación en las convocatorias, así como el desarrollo de los proyectos, se ha convertido en una escuela. De tal suerte que pasados los dos años en los que pueden acceder a la financiación, el $81 \%$ de los grupos continúan realizando las mismas acciones con las que participaron en VAl, o nuevas acciones, pero siguen activos [Secretaría Municipal de Cultura, 2012].

Por esta razón, muchos colectivos se sienten VAl, a pesar de ya no poder participar en las convocatorias y continúan participando de alguna manera en los espacios (muestras culturales] generados por el programa, en las iniciativas ciudadanas que buscan mantenerlo en los momentos de crisis y en los debates que se dieron en el Consejo para la creación de un VAI $2^{10}$. Por otro lado, cada año crece

$9 \quad$ En el año 2012 VAl financió 178 iniciativas culturales de la periferia sobre un total aproximado de 5000 que se han identificado. 10 A comienzos del 2013 comenzaron a darse los debates el número de jóvenes a la espera de los talleres para la realización de proyectos y a la apertura de la convocatoria; en el último año, se presentaron 926 iniciativas. ${ }^{11}$
"VAl trajo nuevas perspectivas, abrió puertas [...] nosotros tenemos una gratitud por la oportunidad de VAl [...] fue un impulso mismo. A partir de ahí vamos a caminar para lograr andar con nuestras propias piernas. Hoy llevamos siete años caminando" [joven de 31 años, convocatoria 2006). [Secretaria Municipal de Cultura, 2012: 80].

Sin duda el público se ha ampliado y esto es determinante en su continuidad, pues si bien es cierto que está reglamentado como ley, esto no impediría que en el momento de una crisis, el programa se reduzca hasta desaparecer. Por esto, el sentido de pertenencia que se ha desarrollado por VAl entre los jóvenes ha sido fundamental para mantener el programa en las diferentes ocasiones en que ha sufrido recortes presupuestales. De alguna manera, los propios jóvenes han enfrentado estas crisis movilizándose y manifestándose frente a la Secretaría de Cultura, consiguiendo con esto descongelar y aumentar los recursos. Y más aún, son las demandas de los colectivos que ya cumplieron su ciclo las que motivaron las discusiones de VAl 2. En el año 2011, el presupuesto presentado por el equipo VAI para financiar 134 proyectos a realizarse en 2012 no fue aceptado. La movilización de la juventud vinculada al programa, a través de Facebook, consiguió un aumento que permitió la entrada de unos 40 proyectos más, llegando a un total de 178.

La primera vez que supimos que los recursos de VAl se iban a congelar y a recortar hicimos una manifestación al frente de la Secretaría; vinieron muchos jóvenes, después de eso, el dinero se liberó. El año 2011 pasó de nuevo, se hizo una convocatoria a través de Facebook, se movilizaron muchas personas, nos manifestamos, estuvimos en el Consejo y justo antes de ir a hacer el plantón frente a la Secretaría dieron la

para la creación de una segunda versión de VAl, que buscaría apoyar ya no iniciativas, sino propuestas más consolidadas. Las convocatorias para participar de estas discusiones son lideradas por movimientos que han pasado por VAl, por los mismos técnicos, por miembros de la Comisión de Evaluación y por jóvenes que buscan participar en el Programa. Nuevamente el Facebook ha sido la herramienta más utilizada para difundir la convocatoria. Ver: https:/ / www.facebook.com/photo.php? fbid=3368189532373\&set=a.1095283111133.201612 6.1498239454\&type=1\&relevant_count=1, https: / www. facebook.com/events/334736006595734/ , https:// www.facebook.com/events/647118425314556/.

11 Recuperado de: http://www.programavai. blogspot.com,el 20 de febrero de 2015. 
noticia de la liberación del dinero. Pero más allá de eso, estas movilizaciones han servido para que como sector nos organicemos y pongamos nuestras demandas, no solo para el presupuesto, sino para que se sepa que están pensando los jóvenes de São Paulo [...] ahora estamos empezando a dar las discusiones sobre la necesidad de un VAI2 [Entrevista con Gabriel di Piero, Red Nossa São Paulo, Grupo de Trabajo de Juventud, São Paulo, octubre 2012.] ${ }^{12}$

En ste sentido, puede decirse que VAl ha sido una escuela de formación para los jóvenes, en la medida en que han aprendido no solo a gestionar y hacer uso de los recursos, sino también a movilizarse como sujetos colectivos para hacer la demanda de sus derechos. Los primeros proyectos presentados eran mucho más individuales y en general enfocados a equipamientos culturales: los jóvenes pedían espacios. Actualmente, los proyectos son articulados, los jóvenes que los presentan piensan mucho más en la circulación y en la apropiación de espacios existentes, en el trabajo en red, en fortalecerse como sector. El proyecto de la Agencia Popular Solano Trindade, es un ejemplo de esto: se trata de un proyecto de red que busca generar circulación y visibilización de diversos colectivos que de alguna manera se ha tomado parte de la zona sur de la ciudad. Una dinámica muy diferente a sus primeros proyectos [Funk concientiza y mujeres periféricas cantan] que se concentraron en hacer producciones

12 Luego de la movilización y liberación de los recursos, el GT de Juventud publicó el siguiente post en su Facebook: "Buenas noticias para todos los productores culturales, grupos y colectivos interesados en la defensa del programa VAl! Después de la movilización intensa, el Secretario Calil anunció la liberación de $\mathrm{R} \$ 900,000.00$ que estaban congelados, gracias a una articulación entre las secretarías municipales [Cultura, Planificación y Relaciones Gubernamentales]. Así, el programa se fortalece y el anuncio de este año puede incluir un mayor número de grupos. Damos las gracias a los grupos, colectivos, artistas y otros ciudadanos que contribuyeron presionando al gobierno! De acuerdo con algunos concejales recibieron más de 100 mensajes de correo electrónico en unos pocos días. Esperamos que este logro abra nuevos diálogos y futuras movilizaciones. El Gt Juventud continúa construyendo una agenda de propuestas de políticas públicas de juventud y enviará una carta a los candidatos de este año, para comprometerlos con nuestras prioridades. Se trata de un espacio autónomo y abierto a la participación, por lo que depende de la contribución de ustedes. Estamos discutiendo temas como el género y la diversidad, centros culturales, VAI2 [para grupos ya cubiertos], la movilidad y el derecho a la ciudad, revisión del papel de los auxiliares de la juventud y regulación de las Estaciones de la Juventud, entre otros temas relevantes. Quién quiera puede traer propuestas, por favor póngase en contacto con nosotros. Saludos generales "GT Red de Jóvenes de Nuestra São Paulo [Facebook Gabriel di Piero: https:/ / www.facebook.com/GabrielDiPierro?fref=ts, 20 de marzo de 2012). [La traducción es mía) musicales con un grupo de personas.

Cuando se hizo la primera evaluación del programa en el año 2008, Helena Abramo, expresó su satisfacción al confirmar la relevancia de esta experiencia pues:

Más allá de crear, efectivamente, la implementación de un canal de acceso al derecho de la producción cultural para jóvenes de la periferia de la ciudad de São Paulo, VAl apunta a principios de directrices que deben ser considerados en el más reciente y creciente debate sobre la formulación de políticas públicas de juventud en nuestro país: la importancia de considerar las demandas de los jóvenes, más allá de los indicadores que los estructuran como grupos vulnerables y en riesgo; la interlocución con los actores juveniles en espacios públicos de formación de las políticas; la definición de ley de programas para jóvenes, en la búsqueda por garantizar su continuidad e inclusión en la planeación estratégica del ejecutivo; la designación de recursos públicos para garantizar los derechos, desde una perspectiva universalizante; la posibilidad de someter las directrices y uso de los recursos a evaluaciones de control social, inclusive de los actores juveniles a los que el programa se dirige.

Más adelante, en la evaluación publicada en el 2012, el Programa se reconoció como parte de las políticas públicas de fomento que atienden las demandas de los movimientos culturales de la periferia, destacándose la importancia que ha tenido para la notable expansión de la escena cultural en la última década, con los 778 proyectos que habían sido desarrollados por jóvenes periféricos en sus ocho años de existencia [2003-2011]. De acuerdo con esta segunda evaluación, uno de los logros más importantes de VAl radica en que potenció la cultura existente en la periferia desde su dimensión artística; es decir, no fue que VAl llevara arte, sino que generó las condiciones para poner en marcha prácticas culturales de mayor calidad, entendiendo que, además de la legitimidad política que tienen las acciones de los colectivos de los barrios periféricos, estas también pueden ser valorizadas desde sus concepciones estéticas. Es decir, el programa VAl ha contribuido en la visibilización de las estéticas de las periferias, revelando un arte y una cultura propios de las llamadas "quebradas", con un fuerte sentido emancipatorio. 
No obstante lo anterior, VAI no deja de ser una política dirigida a valorizar acciones culturales que están alejadas del mercado. Los jóvenes que recurren a este tipo de programas, más allá de buscar una validación desde la cultura y el arte especializados, están buscando en las políticas públicas el reconocimiento y la legitimación de sus acciones y prácticas.

Políticas culturales relativas a la cultura ajena al mercado cultural: se refiere a las formas culturales que, en principio, no se proponen entrar en el circuito del mercado cultural tal como éste es comúnmente caracterizado. En otras palabras, son formas culturales no permeables por el interés económico, tanto en su producción material como en sus objetivos o en la recompensa de sus creadores. Los grupos folclóricos, de cultura popular y de aficionados, constituyen el objetivo por excelencia de estas políticas, que comprenden también los programas orientados a la defensa, conservación y difusión del patrimonio histórico [Coelho, 2009].

Esto no quiere decir que algunos no puedan pasar a ser reconocidos e ingresar en un campo cultural, pero sin duda ya no será más un campo a la manera de Bourdieu en el que se requiere dominar un lenguaje, tener acceso a una buena educación, y a un histórico familiar. Habría que repensar este campo, para ver cómo es que ingresan en los colectivos que no cuentan con ese "acervo" y como es que tiene lugar entonces una otra forma de distinción. Vale la pregunta para el caso de Son Batá, a la luz de su intento por ingresar en el mercado de la industria musical, en un campo cultural que en otro momento les hubiera resultado imposible de penetrar.

Pero antes que los logros alcanzados por los colectivos en términos artísticos, lo que es interesante de una política como VAl, y en general de políticas culturas como las que señala Teixeira Cohelo [2009], es la posibilidad que traen de desencadenar relaciones en la vida de los jóvenes que participan. Relaciones entre los jóvenes y el poder público, entre los jóvenes y la ciudad, y entre los propios jóvenes. Al plantearse como un programa "de fácil acceso, flexible, para personas naturales" VAl está proponiendo de entrada una relación más "tranquila" con los jóvenes; la posibilidad de que los técnicos sean asequibles, de que el equipo acompañe los procesos en todas su etapas, de que exista una oficina donde los atienden en cualquier momento, y de que las personas que hacen parte del Programa como funcionarios, tengan estrechos vínculos con los procesos de las periferias, ha modificado la relación de los jóvenes con el gobierno.
Por otro lado, se ha reconocido la importancia de esta política como apoyo a la creación de redes y la activación de un circuito cultural de la periferia. Entre las redes, se destaca la Agencia Popular Solano Trindade, como una propuesta que busca, además de la articulación, alternativas de sostenibilidad para los diferentes proyectos. El 91\% de los jóvenes entrevistados para realizar el informe entregado en 2012 declararon que sus grupos se articularon con otros grupos [Secretaría de Cultura].

En síntesis, podría decirse que VAl como política pública y como mecanismo de transformación en las formas de actuación de la juventud, ha alcanzado una importante incidencia en la escena cultural periférica de São Paulo que redunda en toda la ciudad. "Cualquier persona que se dedique a investigar sobre la escena cultural de la periferia paulistana, se va a encontrar con VAl. Sea cual sea su foco de análisis tendrá necesariamente que pasar por este programa, pues no es posible hablar de cultura de la periferia sin citar a VAl". [Secretaría Municipal de Cultura, 2012].

\section{Cómo opera VAI}

El programa VAl tiene como objetivo "apoyar financieramente, por medio de subsidio, actividades artístico-culturales, principalmente de jóvenes de estratos bajos y de regiones del municipio desprovistas de recursos y equipamientos culturales" (art. 1, Ley 12.540 del 24 de marzo de 2003- Proyecto de Ley 681 de 2002, del Concejal Nabil Boundiki - PT]. El valor asignado para apoyar cada proyecto varía cada año, yendo en su versión del 2012 hasta $R \$ 23.000$ por iniciativa. Este valor se entrega hasta en tres desembolsos, en un periodo que no puede superar los ocho meses, ni ser inferior a cuatro. Es importante tener en cuenta que VAl busca apoyar iniciativas con notada actuación comunitaria: es decir, se trata de propuestas emergentes, con esto lo que se busca es darles el impulso necesario para que se consoliden y puedan tener una continuidad. Por esta misma razón, la financiación no se extiende a dos periodos, pues se considera que en este tiempo los colectivos alcanzan, de una parte, a aprender a gestionar un proyecto $y$, de otra, a consolidar una propuesta.

El proceso de participación inicia con la inscripción de una iniciativa durante el periodo de convocatoria, que normalmente ocurre en el mes de enero. La única exigencia es haber vivido en la ciudad por un mínimo de dos años y cumplir con el perfil requerido: ser jóvenes de estratos bajos que buscan desarrollar proyectos artístico-culturales. Para la divulgación de la convocatoria se utilizan diversos 
medios, como volantes, plegables, afiches, la página web del programa, redes sociales, voz a voz y se realiza una difusión a través de ONGs, centros culturales, casas de cultura, el Centro de la Juventud, entre otros. Muchos de los proyectos que se presentan vienen de colectivos que se encuentran y realizan actividades, participan en talleres, o son usuarios de estos equipamientos. Esto resulta un poco contradictorio frente a uno de los principios rectores del proyecto, que señala la necesidad de apoyar "regiones desprovistas de recursos y equipamientos culturales"; sin embargo, también es fundamental reconocer estas alianzas como parte del ejercicio de apropiación de los espacios y activación del circuito cultural.

Ahora bien, para inscribirse se debe entregar un proyecto cultural que contenga la información del proponente [generalmente una persona del grupo] y el contenido del proyecto [objetivo, metodología, cronograma y presupuesto, entre otros]. Adicional a esto se debe anexar una ficha que contiene información básica del proponente y del proyecto, que se utiliza especialmente para la sistematización de información y la generación de las estadísticas. Ahora, si bien es cierto que no existe un "formato de proyecto", desde el Programa se ha generado una especie de guía y durante la convocatoria se realizan talleres enfocados a la presentación de proyectos para VAI en diferentes lugares de la periferia, a través de ONGs y centros culturales o de juventud.

Una vez se cierra la convocatoria, las propuestas son evaluadas por la Comisión de Evaluación de acuerdo a unos criterios definidos. Cada proyecto es revisado por dos evaluadores, en caso de empate, los proyectos son discutidos por la comisión en pleno. Una vez son seleccionados, se pasa a un proceso de contratación que implica aportar cierta documentación [cuenta bancaria, verificación de la residencia, etc.] y pasar por un procedimiento un tanto burocrático. Posteriormente, a cada proyecto se le asigna un técnico del equipo. En el año 2012, cada técnico acompañaba alrededor de 30 proyectos en su desarrollo, realización de actividades, presentación de informes y cuentas, reuniones, etc.

Esquemáticamente se puede definir un cronograma del Programa VAl anualmente así: enero: presentación de proyectos; febrero y marzo: evaluación y selección de propuestas; abril: divulgación de los resultados, contratación de los proponentes y encuentros con los grupos; mayo: primer desembolso, inicio de los proyectos con acompañamiento técnico; julio y agosto: primera presentación de cuentas, entrega segundo desembolso; septiembre y octubre: presentación de cuentas del segundo desembolso, entrega del tercer desembolso; prestación de cuentas finales, evaluación con los grupos, llamado para la próxima convocatoria [De Lemos, 2010).
El anterior es un esquema muy general, que puede variar de acuerdo a cada proyecto. Existen, aunque pocos, proyectos de menor cuantía que reciben solo dos desembolsos; por otro lado, a pesar de estipular unas fechas para desembolsos [mayo, julio, octubre], solo el primero se cumple de igual forma para todos; los demás se entregan de acuerdo con la presentación de informes, rendición de cuentas y el cronograma.

Con el propósito de fortalecer el trabajo en red y los circuitos, se realizan tres encuentros: i] previo a recibir el dinero, con el fin de explicar la forma en que se desarrollan los proyectos, ii] al recibir el dinero se reúnen representantes de los colectivos en dos sentidos: por lenguaje y por región, el objetivo es que se conozcan, compartan afinidades, se apoyen en las necesidades e intenten generar redes; $y$ iii) al finalizar el año se hace un encuentro de muestra cultural. Adicional a esto, durante el año se promueven los encuentros por zonas, que son organizados por iniciativa de los grupos con el apoyo de los técnicos que los acompañan. Por su parte, los técnicos son asignados principalmente de acuerdo a afinidades de lenguaje, región o conocimiento del grupo, con el fin de promover relaciones de confianza entre los técnicos y los colectivos; también pueden ser asignados de forma aleatoria.

Durante todo el proceso se hace un especial énfasis en la presentación de cuentas, entendiendo que se trata de recursos públicos y que, si bien la responsabilidad en términos legales recae sobre el proponente que firma el contrato, es una responsabilidad de todo el colectivo. Los jóvenes reciben al iniciar el proceso un "Manual de presentación de cuentas". En realidad, se trata de un proceso sencillo entre el proponente y el técnico: el proponente debe presentar una relación de gastos con las respectivas facturas. De acuerdo con la evaluación del programa desarrollado en 2012 , solo un 10\% de los proyectos presentan graves problemas en la rendición de cuentas o en su desarrollo. Adicional a la rendición de cuentas, se presenta un informe de las actividades en el que es posible, previo acuerdo con el técnico, realizar algunas modificaciones en el cronograma, siempre y cuando estas no sean estructurales del proyecto. Este proceso, además de cumplir con requerimientos formales de la ley y con la rendición de cuentas sobre el gasto de recursos públicos, se constituye en proceso pedagógico de gran importancia para los grupos. VAl es un programa de fácil acceso y desarrollo que sirve como escuela para preparar a los jóvenes para presentarse a convocatorias más exigentes. Muchos de los jóvenes que se presentan a VAl nunca habían hecho un proyecto, no habían llevado la relación de gastos del mismo o un ejercicio administrativo de los diferentes recursos. 
Yo creo que lo más complicado fue la primera vez que presenté un proyecto y aprendí mucho. Al mismo tiempo que fue complicado, creo que fue un periodo de mucho aprendizaje. Las cuestiones del presupuesto, los gastos, ¿sabes?, esa parte burocrática, de todo lo que usted compra tiene que poner la factura. Yo fotocopiaba, guardaba, porque soy organizada; yo estaba muy preocupada. Yo quería dejar todo en orden, todo bien bonito. Pero por ser la primera vez, usted no sabe no cómo hacer las cosas, era como un bicho de siete cabezas [joven que participó en la convocatoria de 2005]. [Secretaría Municipal de Cultura, 2012: 131]

VAl me ayudó mucho a organizar. Los controles financieros que ellos imponen en el proyecto son bien importantes y son una escuela de gestión. Yo creo que fue como un curso de gestión [joven que participó en la convocatoria de 2008). [Secretaría Municipal de Cultura, 2012:130]

El programa prioriza la población joven, entendiendo esta como la que se encuentra entre los 18 y 29 años de edad. Sin embargo, hay que decir que existen excepciones de acuerdo a los proyectos propuestos y se evalúan una serie de factores que pueden permitir vincular a personas mayores. Un ejemplo de esto es el proyecto "Mujeres periféricas cantan", presentado por quienes después serían los fundadores de la Agencia Popular Solano Trindade. Este proyecto vinculaba mujeres adultas y de la tercera edad con mujeres jóvenes, permitiendo un intercambio intergeneracional que fue comprendido por la comisión evaluadora como un elemento muy positivo.

Otra particularidad del programa, de acuerdo con el artículo ocho de la ley, es que permite la participación de un colectivo en dos ocasiones, que pueden ser consecutivas. El objetivo de esto es evitar que ciertos proyectos sean beneficiados "eternamente" y no exista una rotación que permita una participación más amplia del público al cual se dirige el programa. Si tenemos en cuenta que en São Paulo se han identificado a partir de diferentes investigaciones y cartografías cerca de 5.000 colectivos juveniles, y que $V A I$ puede financiar un promedio de 150 por año en los últimos tres años, el programa requiere de estrategias que permitan el mayor acceso posible. No obstante, de acuerdo con los técnicos, hay muchas iniciativas que intentan presentarse una tercera vez, con otro nombre y otro proponente. Para identificar estos casos, ha sido fundamental el conocimiento que han alcanzado los técnicos de cada una de las regiones, así como la memoria que construye la Comisión de evaluación.

Podría decirse que el éxito del programa VAl como política pública municipal está directamente asociado con el pragmatismo que caracteriza sus mecanismos de participación y con la claridad de las reglas del juego [Norberto Bobbio], y esto tiene que ver con el hecho de estar dirigido a un segmento muy específico en unas condiciones de acceso que simplifican la burocracia. En la convocatoria del año 2011 se lee:

El Programa VAl subsidia iniciativas culturales de jóvenes, principalmente aquellos de estratos bajos, con edades entre los 18 y 29 años, que habitan en regiones del municipio de São Paulo con deficiencia de infraestructura y acceso a los equipamientos culturales. Su objetivo es estimular la creación, el acceso, la formación y la participación del pequeño productor y creador en el desarrollo cultural de la ciudad, promover la inclusión cultural y estimular las dinámicas culturales locales y la creación artística en general.

En este sentido, vale la pena preguntarse si el hecho de generar estos dispositivos tan segmentados contribuye realmente a la democratización de la cultura o si, por el contrario, habría que tratar a todos por igual. Pero mientras la inclusión no sea una realidad, es necesario mantener este tipo de políticas; es decir, mientras la oferta del estado no alcance a abarcar a los diferentes sectores poblacionales desde cada campo específico, se hace necesario generar unos mecanismos que garanticen la inclusión de las poblaciones menos favorecidas, como es el caso de los jóvenes de la periferia y esto, sin duda, conduce a segmentar. Ahora bien, si con este tipo de mecanismos se logra visibilizar el sujeto emergente [juventud periférica] a partir del reconocimiento de sus prácticas, ¿cómo pasar al empoderamiento y la emancipación? Porque VAl les permite actuar, ser reconocidos, participar y relacionarse con el Estado, pero de ahí a una verdadera incidencia de estos colectivos hay todavía una gran distancia; esto se sale de los alcances del programa.

\section{Referencias}

Coelho, T. [2009]. Diccionario crítico de política cultural: cultura e imaginario. Barcelona: Gedisa.

Comissão Extraordinaria Permanente da Juventude. [2012]. Brasil. Recuperado de: http:/ / www.camara.sp.gov.br/comissaoextraordinaria-permanente-de-defesa-dos-direitos-da-criancaadolescente-e-dajuventude/.

De Lemos, J. [2010]. “Cultura e política. O caso do Programa VAI em São Paulo 2004-2008”. Tesis para optar al título de Doctor en Ciencias Sociales. Pontificia Universidad Católica de São Paulo.

Secretaría Municipal de Cultura [2008]. VAl - 5 años. São Paulo: Secretaría Municipal de Cultura.

[2012]. Via Vai: percepcoes e caminhos percorridos. São Paulo: Secretaría Municipal de Cultura. 